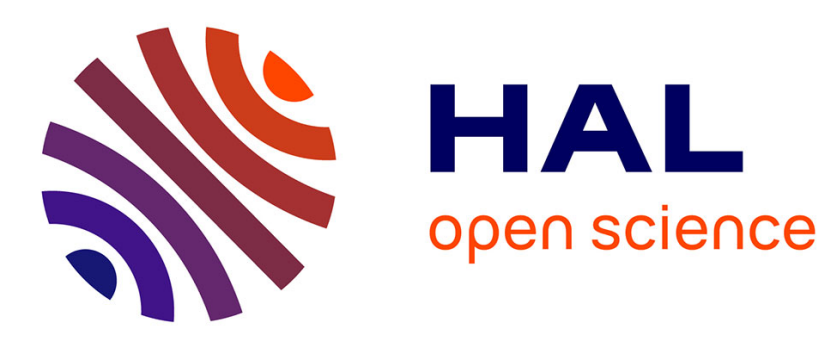

\title{
Prediction of the aerodynamic effect of model deformation during transonic wind tunnel tests
}

\author{
Sylvain Mouton, Marianne Lyonnet, Yves Le Sant
}

\section{To cite this version:}

Sylvain Mouton, Marianne Lyonnet, Yves Le Sant. Prediction of the aerodynamic effect of model deformation during transonic wind tunnel tests. Aerodynamics 2012 (47th AAAF International Symposium of Applied Aerodynamics. Wind tunnel and compulation: a joint strategy for flow prediction), Mar 2012, Paris, France. hal-03017181

\section{HAL Id: hal-03017181 \\ https://hal.science/hal-03017181}

Submitted on 20 Nov 2020

HAL is a multi-disciplinary open access archive for the deposit and dissemination of scientific research documents, whether they are published or not. The documents may come from teaching and research institutions in France or abroad, or from public or private research centers.
L'archive ouverte pluridisciplinaire HAL, est destinée au dépôt et à la diffusion de documents scientifiques de niveau recherche, publiés ou non, émanant des établissements d'enseignement et de recherche français ou étrangers, des laboratoires publics ou privés. 


\title{
Prediction of the Aerodynamic Effect of Model Deformation DURING TRANSONIC WIND TUNNEL TESTS
}

\author{
Sylvain Mouton ${ }^{(1)}$, Marianne Lyonnet ${ }^{(2)}$, Yves Le Sant ${ }^{(3)}$ \\ (1) ONERA - the French Aerospace Lab - F-59045 Lille, sylvain.mouton@ONERA.fr \\ (2) ONERA - the French Aerospace Lab - BP25 F-73500 Modane, marianne.lyonnet@ONERA.fr \\ (3) ONERA - the French Aerospace Lab - F-92190 Meudon, yves.lesant@ONERA.fr
}

\begin{abstract}
During wind tunnel tests, any aircraft model deforms as a result of aerodynamic loads. This behaviour is generally regarded as a spurious effect of the testing technique that needs to be accounted for and corrected. This paper presents available optical technique to measure wing deformation in ONERA wind tunnels, and introduces a simulation method to predict the effect of the deformation on the model aerodynamic characteristics.
\end{abstract}

\section{INTRODUCTION}

Because the internal structure of a wind tunnel model radically differs from that of the real aircraft, the observed deformation in wind tunnel has no similarity property with the deformation of the aircraft in flight. Consequently, the deformation of the wind tunnel model is generally seen as a spurious effect of the testing technique that needs to be accounted for and corrected. As a matter of fact, the observed aerodynamic effect was long expected to be caused by Reynolds effect, while the real cause, wing deformation, had been ignored for a long time. The likely reason is that the deformation level was unknown and barely measurable because of the lack of an appropriate method. Indeed, model deformation was considered a major source of error only recently [1].

This paper focuses on the deformation of the wing of an airplane model, although the method itself could apply to other type of models. The wing of the model is by far the piece that deforms the most because it generates most of the aerodynamic loads and also because of its slender shape. Unfortunately, the wing aerodynamic characteristics are also greatly sensitive to its shape, especially in transonic flow. This problem is even more acute in pressurized wind tunnels.
The complete modelling of the phenomenon would imply to solve a coupled aero-elastic problem, assuming the structural characteristics of the model are well identified. The solution of this problem would yield both the deformed wing shape and the flow-field. This is not the approach presented in this paper. Indeed, the state of deformation of the wing is now systematically measured during most wind tunnel tests, using optical techniques that will be briefly presented. Therefore, we are not interested in determining the deformed wing shape, which is readily available from measurements, but we investigate the effect of this deformation on the aerodynamic characteristics of the wing.

\section{MODEL DEFORMATION MEASUREMENT TECHNIQUE}

There are several methods but the only one that provides real time results is based on marker detection and stereovision which is well known by the Computer Vision (CV) community. Markers are stuck on the model surface which is imaged with two cameras. The Figure 1 shows two typical images as well as a marker. The angle between the two cameras is close to $45^{\circ}$. These cameras (usually more than $2000 \times 2000$ pixels) must have been calibrated previously. The challenge for applications in large facilities is the size of the calibration body. It has to be stiff but must not be too heavy and then cannot be expected without defect. This is why the calibration method includes a tool (known as bundle adjustment by $\mathrm{CV}$ ), which enables to compensate for shape defects.

The basic tool for stereovision is marker detection. The final uncertainty depends on it; this is why a fast and accurate marker detector has been developed. Its uncertainty is of 0.06 pixel which provides a measurement uncertainty lower than $0.1 \mathrm{~mm}$ for a full scale of $1 \mathrm{~m}$. The twist 
uncertainty is lower than $0.05^{\circ}$, even at the wing tip.

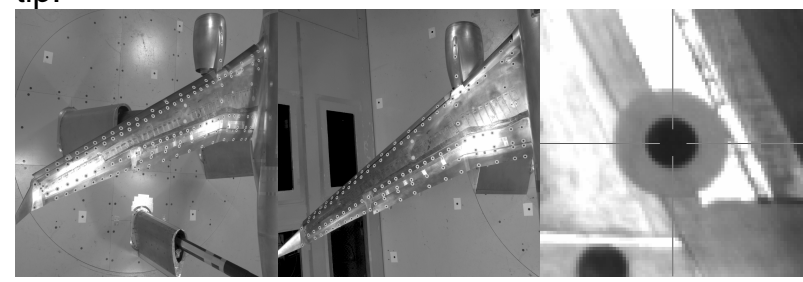

Figure 1: The two images obtained by the two cameras and a marker.

The 3D location of the marker is obtained by using the stereovision principle which is simple: a marker in an image defines a viewing line and the real point is located at the intersection of the two lines. However, they never intersect exactly and there is a small error which is called the epipolar error. The average epipolar error is a good indicator for camera calibration quality. It can be lower than 0.1 pixel and it is considered that it is acceptable for values lower than 0.5 pixel. Greater values mean that the cameras start to be decalibrated, because of their displacement relative to each other.

The ONERA MDM system is routinely available in the largest wind tunnels such as S1MA and F1. It is monitored by the wind tunnel control system as any other usual measurement tool [3]. It can work at a frequency of $1 \mathrm{~Hz}$ mostly limited by the frame rate.

Up to this point, this system could be compared to industrial vision systems and is more an engineering application than a scientific one. However recent developments are scientific matters. The first development is related to the effect of thick windows (several centimetres) or medium change (air/pressurized air) that makes the standard pinhole model not fully reliable. A second one is to track small parts of the wing and to move the cameras to track them when the model moves. The cameras would become decalibrated (only the external parameters) and may be recalibrated minimizing the epipolar error [3]. The effect of air density gradients in the flow around the model (especially through shock waves) was experimentally assessed and found negligible.

The MDM method can be used for other applications than wind tunnel testing. It can be used for conventional shape measurement, for flight testing or for scientific applications that require knowing the object shape with a non contact method. This would be the case for PIV or LDV measurements close to the model surface.

\section{FIRST ORDER FORMULATION}

During wind tunnel tests, data is acquired about the local aerodynamic field (e.g. pressure, temperature, velocity measurements at given points on the model surface or within the flow) and about integral quantities (e.g. forces, mass flow). The local measurements are related to the aerodynamic flow field, represented by a vector of conservative variables $w$. The integral measurements are the result of an integral of $w$ field over some subspace of flow domain (e.g. the skin of the model for forces).

Both the flow field $w$ and the integral $J$ formally depend firstly upon the aerodynamic upstream flow conditions (angle of attack (AoA), Mach number, Reynolds number) and secondly upon the state of deformation of the model wing. We formally regroup the parameters defining upstream conditions under the vector variable $\alpha$, and similarly the deformation state of the wing is supposed to be represented by a vector of $n$ scalar parameters $\theta$ :

$$
\begin{gathered}
J=J(a, t) \\
w=w(x, a, t)
\end{gathered}
$$

The effect of the model deformation $\theta$ at constant $\alpha$ can be expanded as a Taylor series in $\theta$, yielding:

$$
\Delta J(\alpha, \theta)=J(\alpha, \theta)-J(\alpha, 0)=\frac{\partial J}{\partial \theta}^{T} \theta+O\left(\|\theta\|^{2}\right)
$$

The first order effect of the deformation appears as the scalar product of the deformation vector $\theta$ with the sensitivity vector $\frac{\partial J}{\partial \theta}$, i.e. the gradient of $J$ with respect to $\theta$.

To illustrate the application of this, let us consider the three following deformation

- $\theta_{0}$ is a reference deformed shape called the design shape, which generally corresponds to the shape observed under loads corresponding to cruise conditions;

- $\theta_{\mathrm{s}}$ is the shape observed in wind tunnel at given flow conditions

- $\theta_{\mathrm{v}}$ is the shape that would be obtained in flight for the same set of upstream flow conditions

It turns out that:

$$
J\left(\alpha, \theta_{0}\right)=J\left(\alpha, \theta_{s}\right)-\left(\frac{\partial J}{\partial \theta}\left(\alpha, \theta_{0}\right)\right)^{T}\left(\theta_{s}-\theta_{0}\right)+O\left(\left\|\theta_{s}-\theta_{0}\right\|^{2}\right)
$$

When the gradient of the performance $J$ is evaluated for the shape $\theta_{0}$, the performance of the shape $\theta_{0}$ can be obtained from the performance $J\left(\alpha, \theta_{s}\right)$ measured in wind tunnel, with first order accuracy with respect to the distance between design shape and wind tunnel shape. 
Assuming that the flight shape is known, the performance $J$ can be extrapolated to flight thanks to:

$$
J\left(\alpha, \theta_{v}\right)=J\left(\alpha, \theta_{s}\right)+\left(\frac{\partial J}{\partial \theta}\left(\alpha, \theta_{s}\right)\right)^{T}\left(\theta_{v}-\theta_{s}\right)+O\left(\left\|\theta_{v}-\theta_{s}\right\|^{2}\right)
$$

But the sensitivity at $\theta_{s}$ can itself be expanded as:

$$
\frac{\partial J}{\partial \theta}\left(\alpha, \theta_{s}\right)=\frac{\partial J}{\partial \theta}\left(\alpha, \theta_{0}\right)+\left(\frac{\partial^{2} J}{\partial \theta^{2}}\left(\alpha, \theta_{0}\right)\right)\left(\theta_{s}-\theta_{0}\right)+O\left(\left\|\theta_{s}-\theta_{0}\right\|^{2}\right)
$$

which finally yields:

$$
\begin{aligned}
& J\left(\alpha, \theta_{v}\right)=J\left(\alpha, \theta_{s}\right)+\left(\frac{\partial J}{\partial \theta}\left(\alpha, \theta_{0}\right)\right)^{T}\left(\theta_{v}-\theta_{s}\right)+O\left(\left\|\theta_{v}-\theta_{0}\right\|^{2}\right) \\
& +O\left(\left\|\theta_{s}-\theta_{0}\right\|^{2}\right)+O\left(\left\|\theta_{v}-\theta_{s}\right\|^{2}\right)
\end{aligned}
$$

This means that it is possible to extrapolate the wind tunnel performance to the flight performance using the same sensitivity as before, within first order accuracy. The truncation error is made smaller when:

- the chosen reference shape is close from the flight shape and from the wind tunnel shape;

- the wind tunnel shape is close from the flight shape.

Similar formulae can be obtained for the local flow field $w$.

Within the limits of first order accuracy, determining the effect of deformation on the aerodynamic flow field and performance reduces to the determination of their sensitivity with respect to the vector of deformation parameters. Interestingly, once the sensitivity is determined, the calculation of any deformation effect is immediate, meaning they could be accounted for on line during wind tunnel tests.

In this work, RANS equations are solved for the flow around the model to determine performances and the adjoint and linearised methods are used to compute this sensitivity.

\section{PARAMETERISATION}

The above development imposes to represent the displacement of the wing surface under deformation as a finite vector of $n$ scalar parameters.

The slender shape of the wing advocates for the use of beam parameters: bending and torsion at different spanwise sections. Hence, we use in this work the local bending $\delta z$, defined as the displacement in the vertical direction of the point lying at $50 \%$ of local wing chord; and the local aerodynamic torsion $\delta \alpha$ defined as the rotation of the wing section with respect to the span axis. With this definition, it must be mentioned that most of the aerodynamic torsion comes from the bending, since the rotation associated to bending has a projection onto the span axis because of the sweep angle of the wing.

A wing section which displacement and torsion are components of $\theta$ is called a controlled section. Of course, the controlled sections will correspond to the sections were deformation measurement are available from the wind tunnel test. With this parameterization, the twist of one given section has a local effect, mostly limited to the area between current section and its two neighbouring ones.

In the present work, the deformation of both the surface and the volume mesh relied on the free form deformation technique, using direct manipulation [6], but other morphing methods, even analytical ones, would have been efficient as well, provided there are differentiated, as explained in next section.

\section{ADJOINT AND LINEARISED METHODS}

The present work made intensive use of the adjoint and linearised methods to compute sensitivities of solutions of partial differential equation with respect to input parameters. The principles of the methods are not recalled here. A good introduction can be found in reference [5], which gives an overview of its history and present developments. These methods present significant advantages over classical finite difference approach.

It is recalled that the sensitivity of the flow field with respect to each shape parameter is obtained by inverting the linearised equation. For each shape parameter, this implies solving a system of linear equations of very large size, but mostly empty. Once this is done, the sensitivity of any function $J$ is immediately obtained by combination of flow field sensitivities, which permits to evaluate a large number of functions.

In case one is not interested in the sensitivity of the flow field itself, but only in the sensitivity of a small number of functions $J$, the use of adjoint equation method is attractive for cost reason. The adjoint method leads to the inversion of one large size linear system per functions of interest $J$. But the evaluation cost of the sensitivity does no longer depend on the dimension of $\theta$. In the present case, there are few objective functions (typically lift, drag and pitching moment), but a larger number of design parameters (two per controlled wing section).

Both linearised and adjoint equations require complete linearization of the calculation chain to evaluate $J$, from the parameterization to the postprocessing, in order to compute the partial 
derivatives at each stage. In the present work, the parameterization and mesh deformation rely on free form deformation, which is naturally differentiable. Concerning post-processing, the near-field and far-field analyses [2] were also differentiated, using for the far-field an approximation of frozen area of drag production.

\section{VALIDATION CASE}

\subsection{Configuration}

The validation case chosen for this study is a civil transport aircraft configuration called N44. It is composed of a generic fuselage and a wing representative of the design of modern transonic aircraft. The wind tunnel model has been designed so that the wing adopts its design shape (corresponding to aircraft cruise shape) under a lift coefficient of 0.5 and with a dynamic pressure of $88 \mathrm{kPa}$.

Unless one sets up a wind tunnel campaign using several models with different wing deformations or a model deformable on demand, which was never attempted to the author's knowledge, the only way to study the effect of model deformation is to vary the dynamic pressure $q$ of the tunnel. If one demands in the same time that the Reynolds number be constant, not only the pressure but also the temperature of the tunnel should be controlled.

The loads applied to the model are proportional to the dynamic pressure $q$, while the response of the model to the applied loads, i.e. the resulting deformation, is inversely proportional to the Young's modulus E. The shape solution of this aero-elastic problem then depends on the non dimensional ratio $q / E$. Due to the order of magnitude of $q$ and $E$, this ratio is of the order $10^{-6}$. In the following, the $10^{-6}$ is sometimes omitted, and figures are presented in millionth.

\subsection{Experimental database}

This configuration was tested several times in the European Transonic Wind Tunnel ETW [7], both in $1 / 50$ scale full model and in $1 / 30$ scale half model. Test results presented in this paper where obtained in the framework of European projects HiReTT (full model), M-DAW (half-model) and FLIRET (half model) between 2001 and 2007.

ETW is a cryogenic pressurized wind tunnel, enabling the investigation of pure deformation effects by simultaneously varying pressure and temperature. Among the available database, tests at Mach 0.85 and 32.5 millions Reynolds number are presented here. They were carried out for 4 levels of stagnation pressure: 1.6 bars, 2.6 bars,
3.4 bars and 4.4 bars, corresponding respectively to the following $q / E$ ratios: $0.265,0.437,0.565$ and 0.768 millionth.

To avoid introducing bias related to the differences existing between half and full model campaigns, performance comparison is always carried out between data from the same test campaign. One well identified bias is that the "peniche" used to raise the half model out of the boundary layer of the tunnel was included in force measurements, and drag of the half model tests is consequently higher.

\subsection{Wing deformation measurements}

The deformation of the wing was either directly measured or evaluated during the above mentioned campaigns. During HiReTT campaign, the deformation was evaluated thanks to a finite element model calibrated against the real model and on which the loads measured during the test were applied. Various crosschecks not detailed here and carried out by partners of the project lead to a good confidence in the deduced deformation. During subsequent campaigns at ETW, an optical technique similar to the MDM presented in \$2 was available and used. Comparison between the different results reveals a very good agreement in torsion, but a difference in bending that might be attributed to the difference in stiffness of the wing-fuselage junction between full model and half model configurations.

\section{FLOW SIMULATIONS}

\subsection{Direct flow simulations}

Flow simulations were carried out with els $A$ software that solves RANS equations with a finite volume discretization on multiblock structured meshes [4]. The mesh used comprises $3,796,864$ cells and 56 blocks (Figure 2 ).

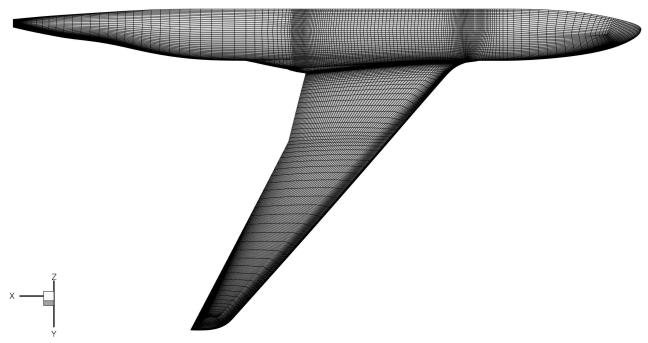

Figure 2: Surface mesh of the model

The simulations presented in this paper used the one-equation Spalart-Allmaras turbulence model for which exhaustive validation is available on similar configurations. Following numerical parameters were used: 
- Roe scheme with Harten entropic correction, extended to second order with MUSCL method, using a Van Albada limiter

- Time marching using a backward Euler method and using implicit formulation solved by a scalar LU-SSOR method

- Convergence acceleration using a 3-level multigrid cycle.

Convergence is obtained after about 800 iterations, but the computation is continued until 1,500 iterations to bring residuals close to machine zero. This indeed contributes to ease the convergence and accuracy of subsequent adjoint computations.

Once the solution flow-field is obtained, it is postprocessed using FFD41 software, which computes the seven force coefficients we are interested in: lift, pitching moment, pressure and friction drag, viscous, wave and induced drag, as well as their derivatives with respect to the flow field and the mesh. For a complete definition of drag breakdown, please refer to reference [2].

\subsection{Adjoint and linearised computations}

The wing deformation is parameterized thanks to 7 controlled wing sections, i.e. 14 scalar parameters. The evaluation of the sensitivities of each function $J$ then leads to 7 adjoint computations or 14 linearised computations.

The adjoint as well as the linearised equations constitute systems of linear equations of very large size, mostly empty and made up of multidiagonal blocks. The resolution method used relies on the same iterative method as the one used in the implicit formulation for time marching during the resolution of the RANS equations, i.e. a LU-SSOR method with four relaxation cycles. At the time of this work, the turbulence model was not differentiated and the adjoint and linearised computations made use of the frozen eddy viscosity hypothesis.

\subsection{Validation of computed gradients}

In order to validate the sensitivities of aerodynamic coefficients computed by adjoint method and linearised method, it is necessary to establish reference values by means of finite difference (FD) method.

Computing an undisputable reference value with FD method is not necessarily an easy task, because the gradient depends on the FD step chosen for each parameter. If the step is too large, second order terms are not negligible, and if it is too small, the difference between computations becomes so tiny that the numerical noise (insufficient convergence, round-off errors) predominates. Therefore, the choice of the FD step needs careful validation.

In this study, the determination of the FD step was carried out on the wing section n $4(y / b=0,611)$. The local twist and bending were varied and resulting variations of the aerodynamic coefficients obtained at Mach 0.85 , at a lift coefficient of 0.5 and around the wing shape for $\mathrm{q} / \mathrm{E}=0.437$ are observed. The gradient of the aerodynamic coefficients is computed using first order and second order FD for different steps of twist $\delta_{\mathrm{DF}} \alpha$ and bending $\delta_{\mathrm{DF}} z$ (in figures rendered non-dimensional using wing local chord $c$ ). Results are presented in Figure 3.

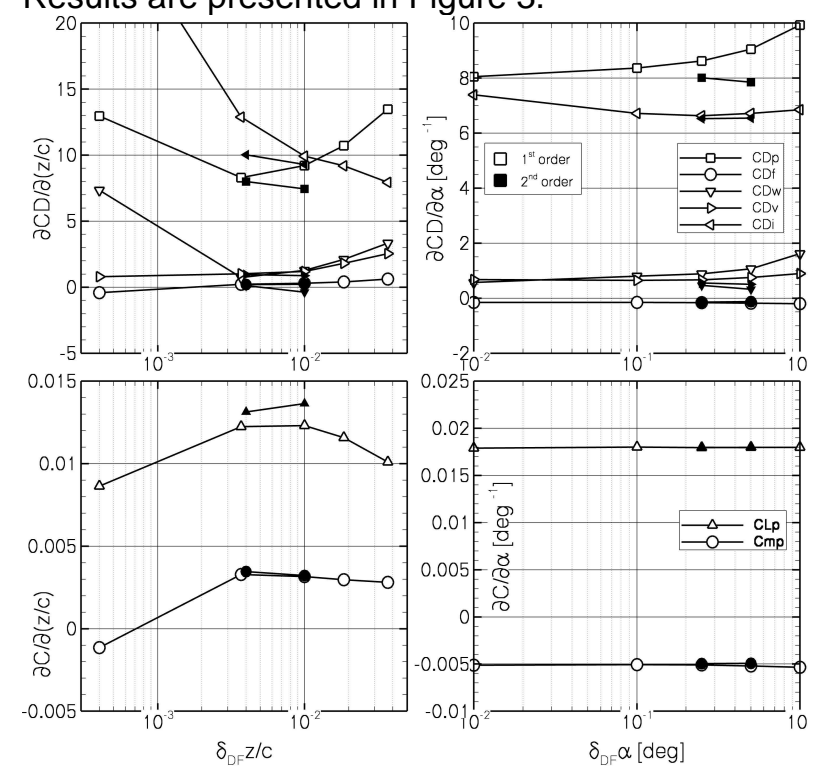

Figure 3: Gradient of different objective functions computed by finite difference on wing section n 4

From this figure, it appears that the sensitivity of some coefficients such as lift is weakly dependent on the FD step. On the contrary, FD estimates of the sensitivity of viscous and wave drag, which behaviour close to the design point is highly nonlinear as will appear later on, are sensitive to the choice of FD step. This is especially true for sensitivity with respect to bending. As expected, second order FD is less subject to this problem.

From these results, for all subsequent FD computations carried out on all wing sections, a step of $1 \%$ of local chord for bending and of $0.01^{\circ}$ for twist were selected, guided by best estimates originating from second order FD.

The gradients obtained by adjoint computations are compared to FD results in Figure 4. The agreement is generally very good, with the exception of pressure drag sensitivity with respect to bending variables. Although the contribution of bending is small compared to the one of twist, this error could not be explained and drag results were 
evaluated using far-field coefficients. Another minor default of the gradient computed by adjoint method lies in the breakdown of the far field drag sensitivity between wave and induced drag. The later is slightly overestimated in disfavour of the former. The results of the linearised method (not shown) were also generally in good agreement with finite difference.
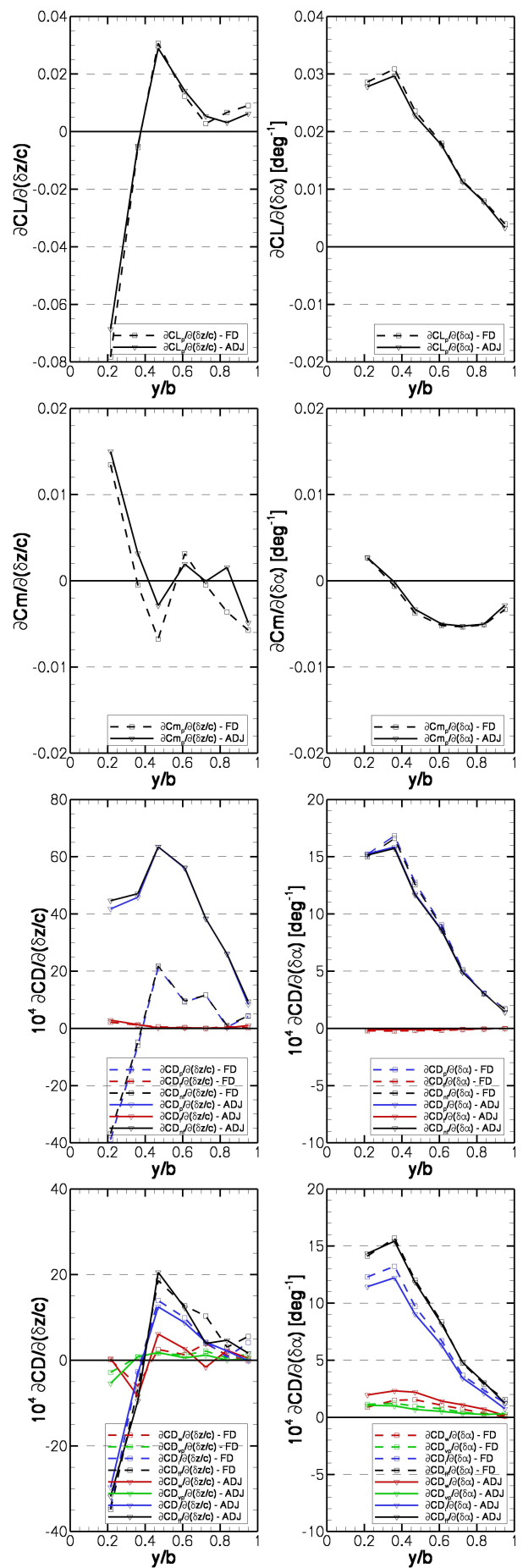

Figure 4: Gradient of lift, pitching moment, nearfield and far-field drag coefficients (from top to bottom) with respect to bending (left) and twist

(right) as a function of position on span of controlled wing section

It is worth mentioning that the contribution of bending to the deformation effect is small in comparison to the twist contribution. On this validation case, it is less than $10 \%$ of the total effect.

\section{EFFECT OF DEFORMATION ON AERODYNAMIC COEFFICIENTS}

\subsection{Effect at cruise point}

The effect of wing deformation at cruise point, corresponding to Mach 0.85 and CL 0.5 was first investigated. Experimental data for wing deformation is available for four dynamic pressures and shown in Figure 5. The deformations presented in this figure are taken with respect to the "wind off" shape. In this work, we used as a reference shape the one obtained at $q / E=0.437$, and deformation are computed with respect to this reference.
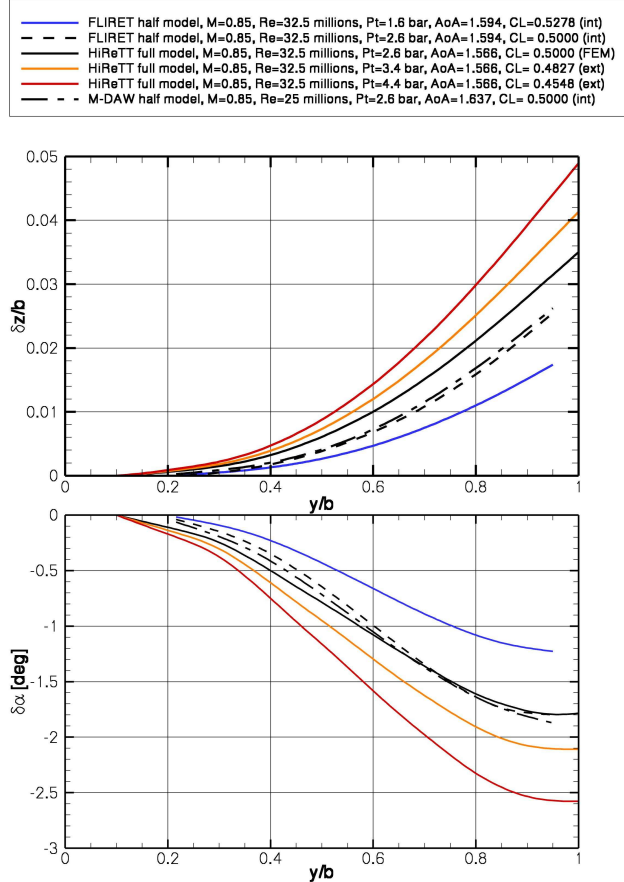

Figure 5: Wing deformation at Mach 0.85 and fixed AoA for several test campaigns

The corresponding flow-field exhibits very weak shock waves. This is a result of careful wing optimization in order to minimize wave drag in cruise.

The effect of deformation on force coefficients was computed by three different methods:

- with several direct computations, i.e. solving RANS equations on deformed meshes; 
- with one direct and several adjoint computations on the mesh corresponding to reference shape;

- with one direct and several linearised computation on the mesh corresponding to reference shape.

The first method yields the exact effect of wing deformation, under the usual restrictions associated with numerical simulations, whereas the last two methods only yield the first order effect of the deformation (see formulae in $\S 3$ ).

Comparison between the different results should be made in two steps:

- comparison between adjoint or linearised computations on the one side and direct computations on deformed meshes on the other side to investigate the magnitude of truncated higher orders in the Taylor development of the deformation effect;

- comparison between direct computations and experiments to characterize the fidelity of the flow simulation.

Such comparisons are carried out in Figure 6. When dynamic pressure is increased keeping all other parameters constant, the load on the model increases. As a consequence of greater lift, the wing bending increases. Mostly because of the sweep of the wing, this bending translates into aerodynamic twist that causes outer wing section to pitch nose down. Therefore, the local lift coefficient of these sections diminishes. Consequently, the lift coefficient of the model also decreases. Because of this change in lift distribution along the span of the swept wing, the nose down pitching moment of the wing is alleviated, i.e. the pitching moment coefficient increases. Finally, the decrease in lift coefficient is associated with a reduction in induced drag, and a variation of wave drag and viscous drag.

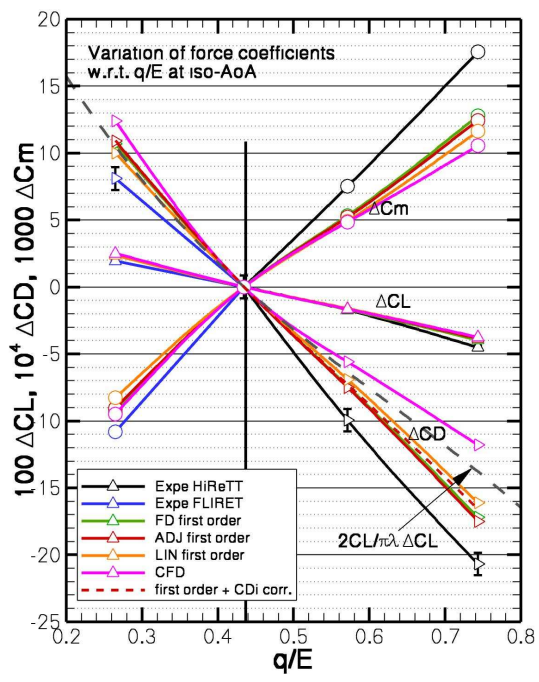

Figure 6: Force increment due to deformation around reference shape at Mach 0.85 and $C L=0.5$

First order increments of force coefficient with respect to $q / E$ are tangent to the complete deviation as expected from the Taylor development. The difference between those two curves represents the higher orders of the development, which magnitude grows when getting farther from the reference shape. Concerning lift and pitching moment coefficient, the higher order terms are negligible over the whole range of deformation under study (which is quite large, covering a twist range of $1.35^{\circ}$ degree at wing tip). On the contrary, higher order terms for drag coefficients represent $40 \%$ of the total deviation ( 6 drag counts) for the larger deformation available. The origin of this behaviour is investigated thanks to the plot of Figure 7.

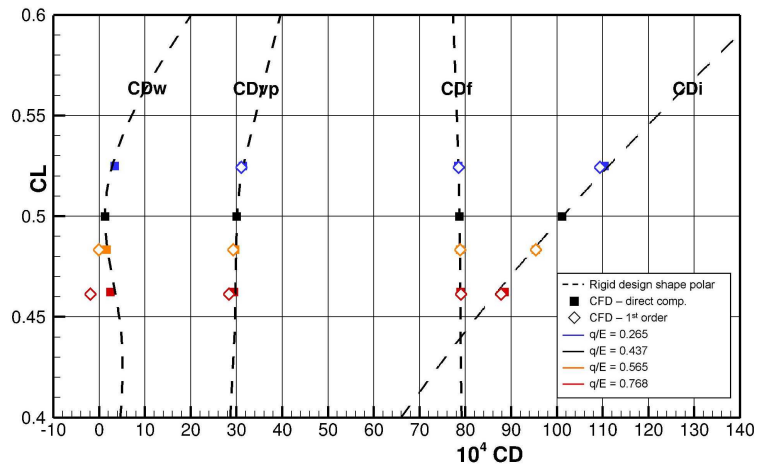

Figure 7: Drag breakdown for different wing shapes at Mach 0.85

This plot presents a drag breakdown of the different computations carried out at Mach 0.85 and a fixed AOA on the shapes corresponding to the four investigated dynamic pressures. It can be seen that the major part of the discrepancy between direct and first order calculations originates from the wave drag. Wave drag behaviour is highly non-linear (with respect to deformation and also to AoA) as a result of wing optimization for cruise. It even presents a local minimum close to the point under study. As a consequence, knowledge of the gradient of wave drag close to this point is of poor interest since higher order terms quickly dominates the results. Consequently, $1^{\text {st }}$ order predictions around the reference shape fall increasingly far from the direct computations when the deformation grows. The same is true to a lesser extent for viscous pressure drag. Finally, induced drag also exhibits slightly non-linear behaviour with respect to deformation, as a response to the lift variations. For the latter, although the first order term already gives a very good approximation of the effect of 
deformation on induced drag, a second order term can be modelled from lifting line results. If one models the induced drag by the classical Prandtl formula:

$$
C D_{i}=\frac{C L^{2}}{\pi \lambda}
$$

Then, variation of induced drag under a deformation writes as:

$$
\begin{aligned}
\Delta C D & =\frac{2}{\pi \lambda} C L \frac{\partial C L}{\partial \theta} \delta \theta+\frac{1}{\pi \lambda}\left(\frac{\partial C L}{\partial \theta} \delta \theta\right)^{2} \\
& =\frac{2}{\pi \lambda} C L \Delta C L+\frac{1}{\pi \lambda} \Delta C L^{2}
\end{aligned}
$$

The second order term (in $\delta \theta^{2}$ or $\Delta C L^{2}$ ) identified thanks to this development can be added to the first order term computed by adjoint / linearised computation. This minor correction brings the results a little closer to direct computations as shown in Figure 6 (curve with label 'CDi corr'). In the same figure, the first order term deduced from this model is plotted for comparison (dashed grey line).

In a second step, the fidelity of the direct calculations with respect to experimental data can be examined in Figure 6. At first sight, this comparison is rather disappointing since curves fall far from each other. However, one can notice that the two wind tunnel campaigns (HiReTT full model and FLIRET half model) yield a very different slope for drag behaviour, with calculations falling somewhere in between. Careful investigations reveal that the shock pattern is different between the two campaigns, with probable consequences on the wave drag behaviour close to the local minimum identified in Figure 7.

Beyond the examination of the force coefficients, the linearised computations also give insight into the effect of the deformation onto the flow field itself, and especially on wing pressure distribution for which exhaustive experimental data is available. The field of pressure sensitivity is displayed in Figure 8. One may note that any deformation in the inner part of the wing has a highly non-local effect that propagates outward to the tip of the wing, even though the deformation itself is local to one wing section. This explains why the effect of those sections on lift is more than proportional to their size.

By taking the scalar product of this sensitivity flow field with the vector of deformation parameters, one obtains the first order effect of the deformation onto pressure distribution. Like for forces, this effect can be compared to direct computations carried out for each deformed shape in order to investigate the magnitude of higher order terms. Then, the direct computations can themselves be compared to experimental data to validate the fidelity of the solution of RANS equations to the experiments.

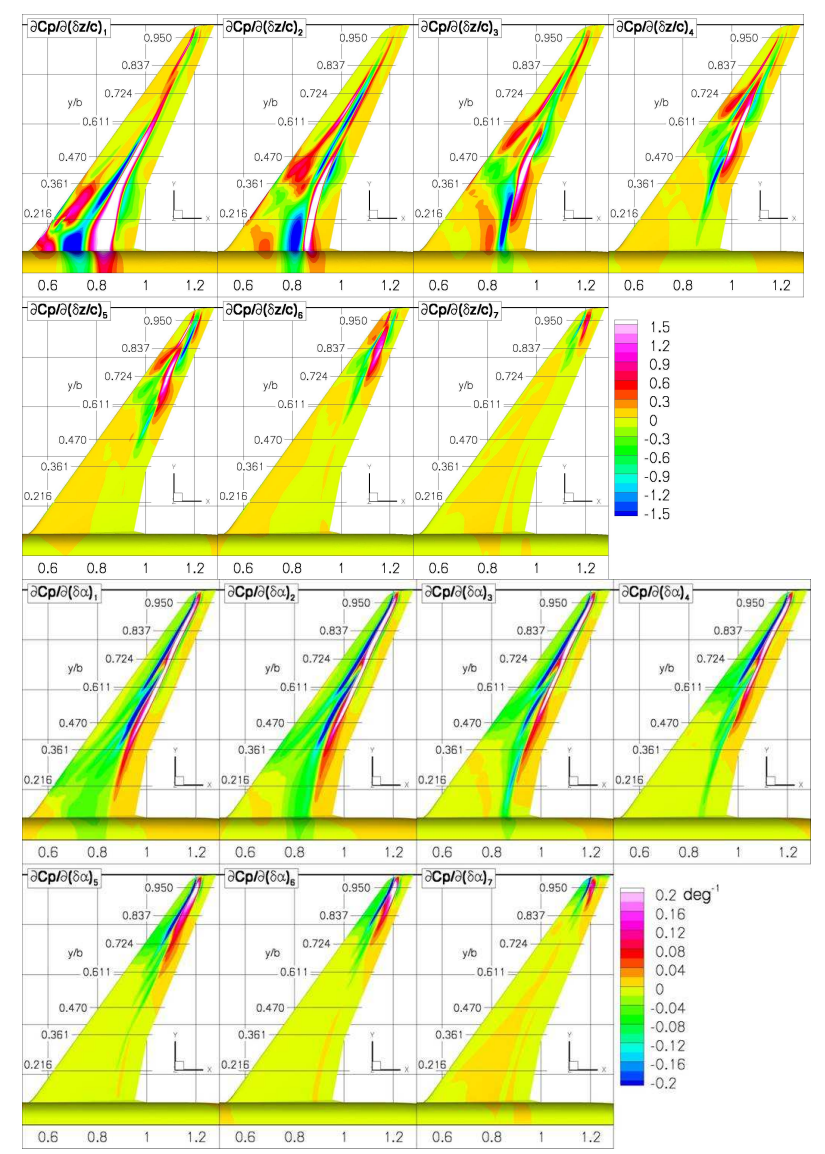

Figure 8: Sensitivity of upper side pressure coefficient with respect to bending parameters (top) and twist parameters (bottom)
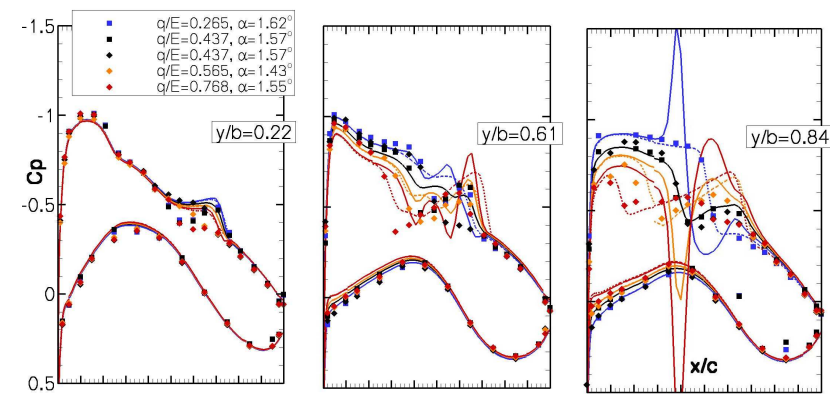

Figure 9: Wing pressure distribution for different wing shapes

symbols: ETW experiments

lines: direct calculations (dashed), linearised calculation (continuous)

Those comparisons, presented in Figure 9, show that direct computations predicts very well the effect of the deformation for most wing sections, meaning the RANS solutions are accurate. This is 
less true concerning the shock behaviour close to mid-span where some discrepancies arise between experiments and calculations, but also between experiments themselves, a phenomenon to be linked to previous remark concerning wave drag.

Concerning linearised computations, apart from the vicinity of shock wave, the prediction is in excellent agreement with direct computations. On the contrary, near the shock waves the discrepancy is considerably large and grows with the magnitude of the deformation. This illustrates again the non-linear behaviour of the flow field around this flight point and the dominance of higher order terms over the first order ones.

\subsection{Effect at low lift coefficient}

The flight point under study in this section corresponds to a Mach number of 0.85 and a lift coefficient of 0.41 . Two dynamic pressure levels are available from experimental data, along with measured deformations. Once again the wing shape obtained at $q / E=0.437$ is chosen as the reference shape, the flow field around this shape is computed and then adjoint and linearised computations are carried out. A direct computation is also carried out around the deformed shape for comparison.

Results are presented in Figure 10 in a way similar to Figure 6. Comparing first order to direct computations, it appears that the first order approach gives prediction that are much closer to direct computations than before, i.e. that higher order terms are much more negligible for this flight point (curves are superimposed on the figure).

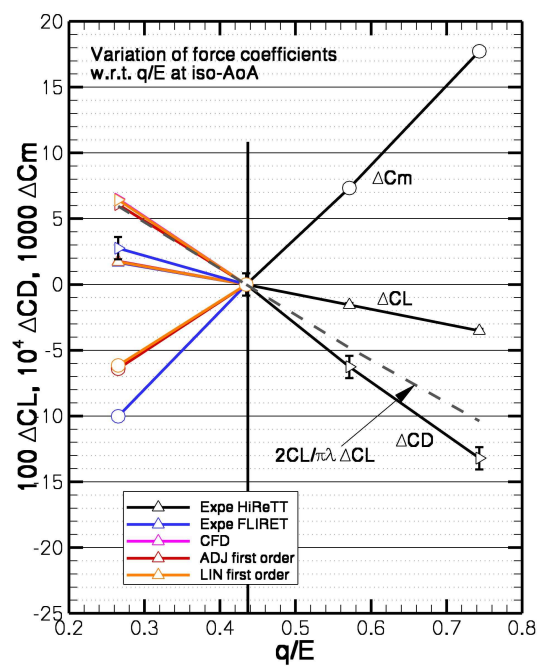

Figure 10: Force increment due to deformation around reference shape at Mach 0.85 and $C L=0.41$
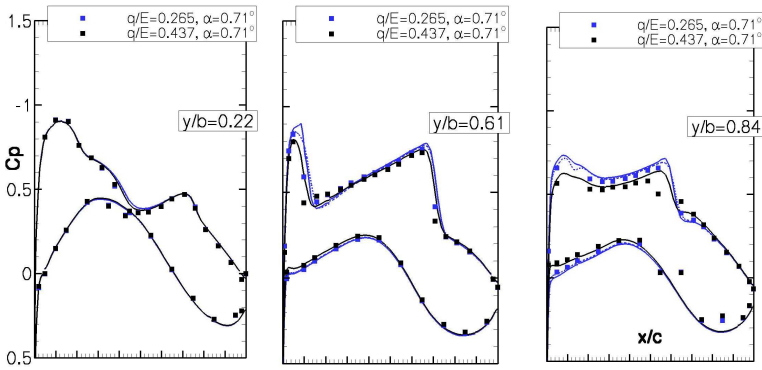

Figure 11: Wing pressure distribution for different wing shapes

symbols: ETW experiments

lines: direct calculations (dashed), linearised calculation (continuous)

Comparison to experimental data shows fair agreement, including on drag coefficient that exhibit large discrepancies in previous section. The only remaining discrepancy in drag is between FLIRET half model test and calculations. If one refers solely to data from HiReTT campaign, the slope of the curve is in very good agreement with computations. For reasons not detailed here, the full model data from HiReTT campaign are considered more reliable. The largest observed discrepancy comes from the pitching moment coefficient for which the effect of the deformation is underestimated by about $35 \%$.

The effect of deformation on the pressure field can now be observed in Figure 10. This agreement between all computations and experimental data is excellent.

\subsection{Effect at high lift coefficient}

The case at Mach 0.85 and CL 0.64 is now examined. This corresponds to a flight point well beyond drag divergence. The shock wave has become so strong that the boundary layer separates at the root of the shock wave over $60 \%$ of span. Consequently, the wave and viscous drag are considerably increased compared to cruise point. On that point, the linearised computations exhibited unsatisfactory convergence. Although they are presented thereafter, they should be interpreted with caution. Once again, the effect of deformation on forces and pressure distribution is presented in Figure 12 and Figure 13. Here again, the agreement between computations and experiment is good to excellent, the only exception concerning the effect on pitching moment, still slightly underestimated. This good agreement appears quite surprising considering the larger viscous effect due to the behaviour of the separated region in response of variations of shock intensity. Indeed the flow separation on the shape at higher dynamic 
pressure has small chord extension (1-2\%), whereas at low dynamic pressure with increased shock intensity, the separation extends over 10 to $30 \%$ of chord. One would expect that such variations be sensitive to a precise modelling of turbulence behaviour and that the frozen eddy viscosity hypothesis would fail in predicting the correct flow sensitivity. Such failure was not observed in the present case.

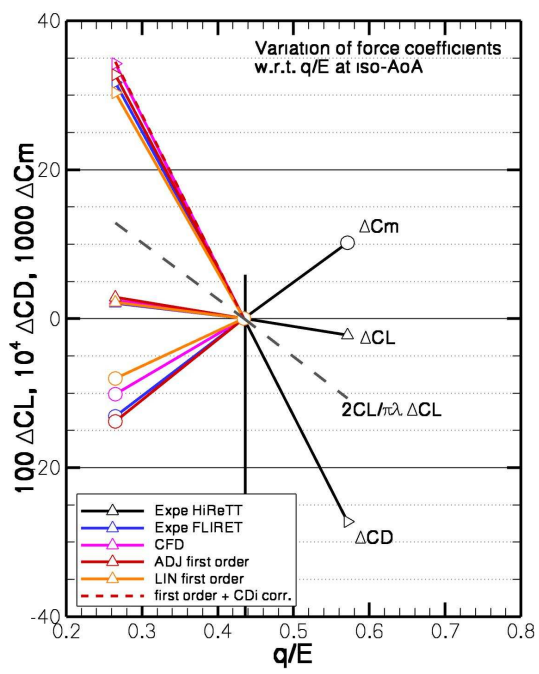

Figure 12: Force increment due to deformation around reference shape at Mach 0.85 and $C L=0.64$
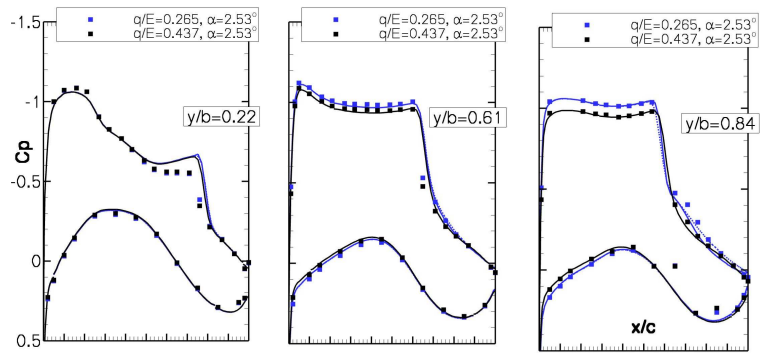

Figure 13: Wing pressure distribution for different wing shape

symbols: ETW experiments

lines: direct calculations (dashed), linearised calculation (continuous)

\subsection{Evolution of gradient with incidence}

As illustrated from the above sections, the gradient of the flow field with respect to wing deformation is a quantity that evolves with angle of attack. This dependency of the gradient with respect to angle of attack is investigated in this section.

Of course, it is not possible to validate against experimental data the evolution of the gradient component by component. Instead, the sensitivity of force coefficients with respect to dynamic pressure is evaluated. For a given coefficient $C_{X}$, this sensitivity writes as:

$$
\frac{\partial C_{X}}{\partial q / E}=\frac{\partial C_{X}}{\partial \theta_{i}} \frac{\partial \theta_{i}}{\partial q / E}
$$

where derivatives are taken at constant flow conditions (AoA, Mach, Reynolds) as before.

Geometrically, this quantity is the slope of the curves showing force increments with respect to $q / E$ in Figure 6, Figure 10 and Figure 12. Experimentally, this sensitivity is evaluated by finite difference between polars at different dynamic pressures. Numerically, the gradient $\frac{\partial C_{X}}{\partial \theta_{i}}$ is the one computed by adjoint/linearised method and the shape sensitivity $\frac{\partial \theta_{i}}{\partial q / E}$ is evaluated by finite difference from experimental data.

The results are plotted in Figure 14 using first and second order finite difference.

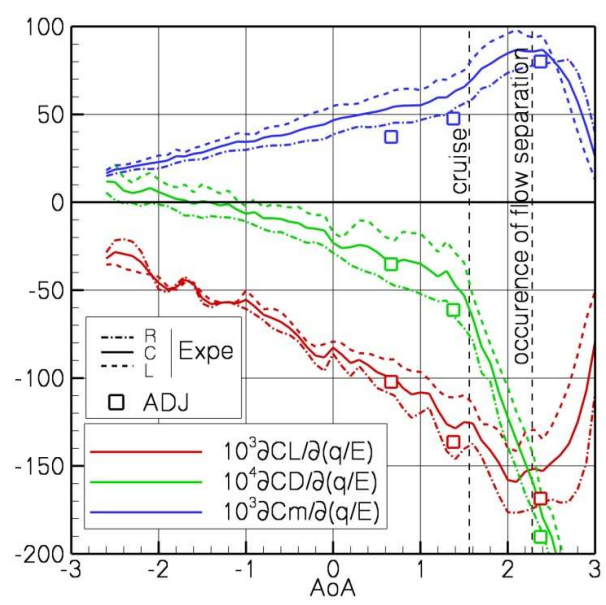

Figure 14 : Evolution with AoA of force sensitivities to wind deformation caused by a change in dynamic pressure at iso-AoA and around $q / E=0,437$

Symbols: simulations by adjoint method Lines: ETW experiments (continous: centered $2^{\text {nd }}$ order $F D$, dashed: right $(R)$ or left ( $L$ ) first-order $F D$ )

In this figure, experimental data obtained using right-side finite difference are probably the most reliable since other data (left side and centered) relies on measurement from the half model campaign which, as previously mentioned, exhibits certain incoherencies. It can be said that the discrepancies between numerical and experimental data are close to the experimental uncertainty. The evolution of the shape sensitivity 
with the AoA is well predicted by numerical simulations, including beyond the drag divergence where its behaviour radically changes.

\section{CONCLUSION}

Adjoint and linearised computations on a civil transport aircraft allowed the prediction of the sensitivity of force coefficients with respect to the wing shape. The calculations were validated against direct simulations of the deformed shape and against available experimental data from cryogenic wind tunnel, exhibiting reasonable agreement. Thanks to MDM measurements technique also presented, the deformed wing shape is known during wind tunnel tests. Such computations may then be used to extrapolate wind tunnel test results to the design shape (infinitely rigid aircraft) or even to any other known shape (like flight shape for example). The extrapolation is accurate to the first order, which was shown to be sufficient for most of the polar, but presented some shortage close to the design point where the flow field behaviour, especially the shock wave, is far from linear.

\section{REFERENCES}

1. Liu, T., Radeztsky, R., Garg, S., Cattafesta, L. (1999).

A Videogrammetric Model Deformation System and its Integration with Pressure Paint. $37^{\text {th }}$ AIAA Aerospace Sciences Meeting and Exhibit, AIAA 99-0568, Reno, January 11-14, 1999.

2. Destarac, D. (2003).

Far-Field / Near-Field Drag Balance

Applications of Drag Extraction in CFD. VKI Lecture Series.

3. Le Sant, Y., Mignosi, A., Touron, G., Deléglise, B., Bourguignon, G. (2007). Model Deformation Measurement (MDM) at ONERA. $25^{\text {th }}$ AIAA Applied Aerodynamics Conference, AIAA 2007-3817, Miami, 25-28 June 2007

4. Cambier, L., Veuillot J.-P. (2008).

Status of the elsA CFD Software for Flow Simulation and Multidisciplinary Applications, $26^{\text {th }}$ AIAA Applied Aerodynamics Conference, AIAA 2008-664, Reno, January 7-10, 2008.

5. Peter, J., Dwight, R. (2010). Numerical Sensitivity Analysis for Aerodynamic Optimization: a Survey of Approaches, Computers and Fluids, Vol. 39, Issue 3, pp 373-391.
6. Yamazaki, W., Mouton, S., Carrier, G. (2010). Geometry Parameterization and Computational Mesh Deformation by Physics-Based Direct Manipulation Approaches. AIAA Journal, Vol. 48, No. 8.

7. Green, J., Quest J. (2011). A short history of the European Transonic Wind Tunnel ETW, Progress in Aerospace Sciences, Vol. 47, Issue 5, pp 319-368. 